\title{
Joint Discussion 13 Exploiting large surveys for Galactic astronomy
}

\author{
Christopher J. Corbally ${ }^{1}$, Coryn A.L. Bailer-Jones ${ }^{2}$, \\ Sunetra Giridhar ${ }^{3}$, and Thomas H. Lloyd Evans ${ }^{4}$ (eds.) \\ ${ }^{1}$ Vatican Observatory Group, University of Arizona, Tucson AZ 85721, USA \\ email:corbally@as.arizona.edu \\ ${ }^{2}$ Max-Planck-Institut für Astronomie, Königstuhl 17, D-69117 Heidelberg, Germany \\ email: calj@mpia.de \\ ${ }^{3}$ Indian Institute of Astrophysics, Koramangala, Bangalore 560034, India \\ email: giridhar@iiap.res.in \\ ${ }^{4}$ SUPA, School of Physics and Astronomy, University of St Andrews, \\ North Haugh, St Andrews, Fife KY16 9SS, UK \\ email: thhle@st-andrews.ac.uk
}

\begin{abstract}
This summary of JD13, Exploiting large surveys for Galactic astronomy, is based on the talks that were given during the meeting and/or submitted to the full proceedings which appeared in MemSAI 77 no. 4, 2006. The electronic version of this MemSAI volume also has the abstracts and images of the posters that were displayed and discussed during JD13. Here can only be listed their titles and authors. A panel discussion followed the talks, and a summary of the topics covered is added. Finally, there come the concluding remarks.
\end{abstract}

Keywords. surveys, Galaxy: fundamental parameters, stars: fundamental parameters, techniques: miscellaneous

\section{Preface}

While surveys have always been the lifeblood of astronomy, the current era has seen an explosion in both quantity and quality of survey data, made possible by digital instrumentation and electronic databases. Since these surveys are using immense resources, it seemed important to pose the question of how to get the best out of them. This IAU Joint Discussion 13 concentrated particularly on those surveys whose targets are stars and so bear on astrophysics within the Milky Way and Local Group. Time limitations dictated emphasis on the optical and near-infrared surveys, but without excluding the higher and lower energy surveys.

The JD13 started by reviewing the major surveys to find out what these were telling us about the formation and evolution of our Galaxy, the model of an average late-type spiral galaxy. It looked carefully at the techniques of photometric and spectroscopic classification used to identify stars of different types, at stellar variability for distances, and at the complementary kinematic data from radial velocities and proper motions. The relationship of these data with theoretical models of stellar structure and atmospheres was then considered, for these models are critical to age determinations and parameterization. 
Our meeting highlighted the remarkable achievements of large surveys, but it also provided a forum in which participants could discuss and reflect on how far the analysis methods were achieving what was hoped from these surveys and so how best to exploit future surveys. The opportunity given at the IAU General Assembly XXVI in Prague for a gathering of astronomers from many fields proved ideal, if short, for promoting this fruitful exchange of expertise and ideas.

Individual contributions from the authors of the eighteen invited talks and the thirtynine posters, the panel discussion, and the concluding remarks, all of which composed our day and a half meeting, were beyond the page limit set for JD13 in these Highlights of Astronomy. Instead, you will find in the following pages summaries. These were written by a few of those closely involved in the conception and execution of this meeting's topic and were based on the submitted papers. For the full papers of JD13, authored by the individual speakers, and for viewing the very attractive posters, with updated abstracts, please consult the Memorie della Società Astronomica Italiana, vol. 77, no. 4, at <http://sait.oats.inaf.it>

It is our great pleasure to acknowledge the IAU Secretariat and Executive Committee for grants that made the attendance of some speakers and key participants possible. We also warmly thank the IAU-GA XXVI's most attentive Local Organizing Committee.

\section{Scientific Organizing Committee}

Further, this JD13 would not have been possible without the expertise and active involvement of all the Organizing Committee members:

Coryn A.L. Bailer-Jones (Germany, co-chair), Christopher J. Corbally (Vatican City, cochair), Laurent Eyer (Switzerland), Sunetra Giridhar (India, co-chair), Thomas H. Lloyd Evans (U.K.), Dante Minniti (Chile), Heather L. Morrison (USA), Birgitta Nordström (Denmark), Imants Platais (USA), and Patricia A. Whitelock (South Africa).

It was a pleasure to work with them all and together achieve a meeting that was enjoyable at the time and will hopefully prove of lasting benefit to Galactic astronomy.

We look forward to the future surveys and to our further reflections on them.

Christopher Corbally, Coryn Bailer-Jones, and Sunetra Giridhar, co-chairs SOC, and Thomas Lloyd Evans, co-author of proceedings.

Tucson, Arizona, USA, October 31, 2006 


\section{Photometric, spectroscopic and kinematic Surveys} Reported by Thomas H. Lloyd Evans

\subsection{Rosemary F.G. Wyse: Lessons from surveys of the Galaxy}

Wide-field imagers and multi-object spectroscopy, from the ground and increasingly from space, permit large surveys of stars in galaxies of the Local Group. These may be compared to the predictions of Galaxy formation and to the results of high-redshift surveys on the properties of galaxies in the early Universe. Early surveys, which comprised mainly photographic photometry in a few pass-bands, sufficed to give an outline of the galactic halo and the thick and thin disks. Spectroscopy of relatively small samples gave an indication of the metal contents of these subsystems and permitted the estimation of some dynamical properties. Distance estimates and proper motions together then enable samples to be plotted in phase space. Much subsequent work was guided by the ELS model of the formation of the Galaxy. Stars of high proper motion were used to trace the halo and thick disk. The work of Tinsley stressed the need to understand the evolution of the Milky Way, a typical galaxy, as a precondition for understanding the Hubble diagram.

The current paradigm of galaxy formation by mergers, instead of the collapse of a single cloud postulated by ELS, introduces new considerations. The orbital energy of the merging galaxies is converted into internal degrees of freedom. The low density outer regions of the smaller systems are removed by tides and the thin disks are heated, a permanent consequence for the stars though not for the gas. The outer parts gain angular momentum at the expense of the inner parts, so gas and stars are driven towards the centre and any disk formed later will have a short scale-length. Predictions include the late formation of the extended thin disk, after merging is largely completed, and the formation of the stellar halo from disrupted satellites. The extent of change depends on the relative masses involved: collision with a galaxy with less than $20 \%$ of the mass of the disk will heat the thin disk to create a thick disk and add material to the bulge, while a more significant merger may transform a disk galaxy to an S0 or an elliptical galaxy. The disk may be re-created by subsequent accretion of gas and possibly stars. Many stars in the solar neighbourhood formed at redshifts greater than 2 , or an age $\geqslant 10 \mathrm{Gyr}$, and retain memories of conditions at these early times, thus enabling a local approach to cosmology.

The thin disk has an exponential structure with scale-length of $3 \mathrm{kpc}$ and scale-height of older stars of $300 \mathrm{pc}$, with a mass around $6 \times 10^{10} \mathrm{M}_{\odot}$. Observations of nearby stars indicate an approximately constant star formation rate with low-amplitude bursts every few Gyr. The mean metallicity is close to solar and there is not a close correlation of age and metal content. The presence of very old thin disk stars at the solar circle, or 3 scale-lengths, is hard to explain in the $\Lambda$ CDM model, hence the suggestion that they have been accreted. Many galaxies with prominent extended disks have been observed at redshifts of 2 and greater. The old stars in the local thin disk formed at a redshift $z>1.5$.

The thick disk has a scale length of $3 \mathrm{kpc}$ and a scale-height of $1 \mathrm{kpc}$ and accounts for about $5 \%$ of the local density. The mean metallicity is -0.6 dex and the age is similar to those of globular clusters of similar metallicity, 10-12 Gyr. The elemental abundance pattern is distinctive. The thick disk lags the thin disk by $30-50 \mathrm{~km} / \mathrm{s}$ and has a vertical dispersion of $45 \mathrm{~km} / \mathrm{s}$, which is hotter than would be expected from heating by disk perturbations such as giant molecular clouds and spiral arms. The predominantly old age argues against extended heating of the thin disk, while if it was merger-induced, the last significant merger was surprisingly long ago, at redshift about 2 . The high metallicity 
is difficult to account for if the bulk of the thick disk is from accreted satellites. The Milky Way is not unusual in possessing a thick disk.

The Bulge has exponential scale-length of $500 \mathrm{pc}$, scale-height of $300 \mathrm{pc}$ and is mildly triaxial with a bar; the mass is $10^{10} \mathrm{M}_{\odot}$. The mean metallicity is below solar and the dominant age is old. The few elemental abundances which have been measured suggest enrichment by Type II supernovae, which suggests a short duration of star formation. The IMF of low mass stars is the same as that seen in metal-poor globular clusters, the local disk and in the UMi dwarf spheroidal (dSph) galaxy.

The stellar halo within $15 \mathrm{kpc}$ of the Galactic Centre has a total mass of $10^{9} \mathrm{M}_{\odot}$ and is flattened by $\mathrm{c} / \mathrm{a}$ of about 0.5 at the solar distance. It comprises mainly old, metalpoor stars in orbits of low angular momentum. These are very different from those in dwarf galaxies now, so late accretion has not been important. There is remarkably little scatter in elemental abundance ratios, with a flat 'Type II plateau' at all metallicities: this is unlike the situation in dwarf galaxies, and provides no evidence for changes in the massive star IMF.

The outer halo, with dynamical timescales in excess of $1 \mathrm{Gyr}$, is the best place to find structure, and several streams have been found in both coordinate space and kinematics. The Sagittarius dwarf galaxy accounts for much of this, with streams around the sky detected in 2MASS photometry. SDSS data have recently revealed a narrow 'orphan' stream, as well as a Monoceros stream which may indicate disk accretion. There is a stream associated with the globular cluster Pal 5, but this results from star loss by the cluster and not accretion by the Galaxy. A component of the thick disk, identified in faint F/G stars at several kpc above the Galactic Plane, is characterised by low angular momentum and metallicity and may represent a shredded satellite galaxy. The same data reveal a possible retrograde stream. Moving groups and stellar streams within the thin disk may reflect past disturbances by spiral arms, rather than stars accreted from dwarf galaxies. The new surveys have sufficient depth to include stars in the dwarf galaxies which surround the Milky Way. SDSS data have revealed additional dSph galaxies in Boo and CVn; radial velocities confirm the reality and delineate the extent of the Boo dSph.

Large surveys are needed to quantify both small-scale and large-scale structures. Excellent photometry, positions and proper motions are required to analyse spatial structure in colour space. Stars need to be selected carefully for spectroscopy of medium resolution, to give kinematics and metallicity, while a subset must be observed at high resolution to provide elemental abundances. Several large scale spectroscopic surveys are under way.

\subsection{Heidi J. Newberg: Galactic structure from photometric surveys}

Photometric surveys are the most effective way to study large scale Galactic structure, at least until radial velocity surveys on a similar scale are available. Photometric criteria permit the selection of specific types of star: examples are BHB stars, RR Lyrae stars, F-type turnoff stars, M- and K-type giants. The luminosities of these stars are sufficiently well known to permit their use as tracers of galactic structure. Results for the smooth components of the Galactic spheroid and disk structures are not in good agreement with one another, perhaps because discrete structure within each component has not been accounted for. Published results do not agree to within the respective errors: K-type giants selected from 2MASS gave scale heights of the thin and thick disks of $269 \pm 13 \mathrm{pc}$ and $1062 \pm 52 \mathrm{pc}$, respectively; main sequence stars with SDSS data gave $330 \pm 3 \mathrm{pc}$ and $580-750 \mathrm{pc}$ in one investigation and $280 \mathrm{pc}$ and $1200 \mathrm{pc}$ in another. These discrepancies are unlikely to be attributable to uncertain luminosity estimates, as the results for the 
thin and thick disks are not in the same ratio in the different studies. It may be that the situation is confused by structure additional to the large scale structure envisaged.

Seventeen of the twenty dwarf galaxies known to surround the Milky Way have been found by photometry. Most recent discoveries have been made with SDSS data and one, the possible example in Canis Major, from 2MASS. The latest discoveries are all of very low surface brightness and it is difficult to distinguish between dwarf galaxies and star clusters. These objects are detected as statistically significant excesses of star counts at a two-dimensional location in the sky, and locality in distance is determined from a colourmagnitude diagram in which features such as the main sequence, horizontal branch and giant branch may be recognised.

At least six tidal debris streams, which extend over tens of degrees of sky, have been identified, five of them in SDSS data. Three may be associated with dwarf galaxies and three are probably associated with globular clusters. Five of them were discovered in the SDSS data by convolving a template colour-magnitude density profile with the data. The Sgr dwarf galaxy was detected using carbon stars as tracers, but the associated stream was detected spatially with A star tracers from the SDSS, and later shown to extend all the way around the sky using stellar tracers from the SDSS and from 2MASS. These dominate photometric surveys of much of the sky, so that no photometric survey of a small area could measure the parameters of the spheroid reliably.

The spheroid is not axially symmetric but may be fitted by a triaxial profile. An overdensity in Virgo, apparent in studies of A and F stars, makes a large contribution to this asymmetry, which is therefore hard to establish. The Virgo overdensity itself overlaps both the leading and the trailing Sgr tidal tails, but this structure is receding and is unlikely to be associated with the infalling leading tidal tail of Sgr, while the Virgo stars are redder than those typical of the Sgr tail. It is widely separated from the trailing Sgr debris and in any case it appears to have a centre, unlike the tidal tails. Some of the BHB stars in the photometric survey have measured radial velocities. The resulting picture is complex, but there is a significant positive peak in velocities near the centre of the Virgo overdensity, consistent with the finding from RR Lyrae stars.

\section{3. Željko Ivezić: The SDSS spectroscopic survey of stars}

The Milky Way is usually modeled by three components: the thin disk, with $\sigma_{\mathrm{z}} \simeq 20 \mathrm{~km} / \mathrm{s}$ and a scale height of $\sim 300 \mathrm{pc}$, the thick disk with $\sigma_{\mathrm{z}} \simeq 40 \mathrm{~km} / \mathrm{s}$ and a scale height of $\sim 1 \mathrm{kpc}$ and a lower average metallicity $\left(\left[Z / \mathrm{Z}_{\odot}\right] \simeq-0.6\right)$, while the halo has low metallicity $\left(\left[Z / \mathrm{Z}_{\odot}\right]<-1.5\right)$ and little or no net rotation. A full description of the Milky Way requires knowledge of the distributions of the three spatial coordinates, three velocity components and metallicity.

The SDSS provides photometry and spectroscopy for stars in a quarter of the celestial sphere in the North Galactic Cap. Flux densities are measured in $u$, with effective wavelength $3540 \AA$ and limiting magnitude 22.1, $g(4760 \AA, 22.4), r(6280 \AA, 22.1), i(7690 \AA$, 21.2) and $z(9250 \AA, 20.3)$. A total of 100 million stars in $10000 \mathrm{deg}^{2}$ will be observed, with completeness dropping from $99.3 \%$ at the bright end to $95 \%$ at the faint limits quoted. The RMS photometric accuracy is $0.02 \mathrm{mag}$ at the bright end and the absolute zero point calibration is accurate to within about $0.02 \mathrm{mag}$. The astrometric positions are accurate to about 0.1 arcsec in each co-ordinate for stars brighter than $r \simeq 20.5$ mag, and star-galaxy separation is reliable to $r \simeq 21.5 \mathrm{mag}$.

Spectra for over 150000 stars in the North Galactic Cap have been obtained with a spectroscopic resolution of 2000 over the range 3800 - $9200 \AA$. Spectral types and radial velocities are determined by matching the measured spectrum to a set of templates, which are calibrated using the ELODIE stellar library. Random errors in the velocities 
depend on spectral type but are usually $<5 \mathrm{~km} / \mathrm{s}$ for stars brighter than $g \simeq 18$, rising to $\sim 25 \mathrm{~km} / \mathrm{s}$ for stars with $g=20$. Multiple observations suggest that these errors are underestimated by a factor of $\sim 1.5$.

Sixteen stars of spectral type near F8 were selected on each plate as spectroscopic standards to calibrate the spectrophotometry. The spectra were used to estimate the effective temperature, gravity and metallicity of the stars observed. Linear relationships were established between effective temperature and $g-r$ and between metallicity and $u-g$, in a limited range of colour in each case. Errors of $100-200 \mathrm{~K}$ in $T_{\text {eff }}$ for stars in the $-0.3<(g-r)<1.0$ colour range and 0.3 dex in metallicity for stars at the blue tip of the stellar locus, $(u-g)<1$, have been attained. These relationships were then used to determine effective temperature and metallicity for the much larger number of stars for which only photometric data were obtained.

Metallicity estimates were then made for a sample of 10000 blue main-sequence stars with $14.5<g<19.5,0.7<(u-g)<2.0$, and $0.25<(g-r)<0.35$. The last condition selects stars of temperature $6000-6500 \mathrm{~K}$, with an additional restriction on distance from the locus in the $(g-r)$ vs. $(u-g)$ plane to confine the sample to the main stellar locus. The metallicity distribution of stars a few kpc from the galactic plane is bimodal, with a local minimum near $[\mathrm{Fe} / \mathrm{H}]=-1.3$. The stars of higher metallicity have a much larger density gradient in the $z$ direction than in the $R$ direction, whereas the stars of lower metallicity show little dependence on position within $4 \mathrm{kpc}$ of the Sun. The high metallicity stars presumably represent the thin and thick disks, but there is no indication of the existence of two distinct groups in these data. Radial velocity data similarly show no such distinction. The stars of low metallicity have a velocity dispersion which is about 2.5 times higher than that of those of high metallicity, extending this well-known correlation to a much larger volume of space. Stars of high metallicity in an anticentre field show a higher velocity dispersion and an anomalous rotational velocity, possibly indicating the presence of a stellar stream.

\subsection{Johan Holmberg: Revisiting the Geneva-Copenhagen Survey}

The Geneva-Copenhagen Survey (GCS) provides metallicities, ages, kinematics and Galactic orbits for $14000 \mathrm{~F}$ - and G-type dwarfs with $V \leqslant 8.3$. These were based on uvby photometry, Hipparcos/Tycho-2 parallaxes and proper motions, and new accurate radial velocities. The GCS provides large samples of stars over a full range of age, metallicity and abundance, while it is largely free of selection by kinematics or metallicity. The transformations from observational data to astrophysical parameters may introduce systematic errors and this paper re-investigates the underlying calibrations.

The GCS obtains effective temperatures from a calibration of $b-y, m_{1}$ and $c_{1}$. The availability of the 2MASS photometry permits a calibration based instead on $V-K$, which appears to give more consistent results. There is less systematic error, but the observational error of the $b-y$ index is less than that in $V-K$, so the $V-K$ temperature scale is used to provide a new calibration of $\mathrm{T}_{\text {eff }}$ as a function of $b-y$. The resulting calibration recovers the correct effective temperature of the Sun, and has a dispersion of $60 \mathrm{~K}$.

The GCS used a composite metallicity scale which was based on several separate calibrations. Nearly 600 stars, which have recent abundance determinations based on a correct temperature scale, have been used to relate the $u v b y$ indices to metallicity. The redder and bluer stars are still calibrated with the earlier relations. The Hyades presents a particular problem, because the published photometry is not on the same system as that used for the other stars, and because the cluster has an unusual He/Fe ratio. It cannot therefore be used to check the age and metallicity scales for field stars. Ages and 
masses depend on isochrones in the CM diagram, and these need to be revised using the new temperature and metallicity calibrations. Wide physical binaries provide a check on the calibrations, and differences in age and metallicity of the components are reduced with the new calibrations. The new calibration of age is systematically different from that of the GCS: the greatest ages are reduced by about $10 \%$. Incorrect results are still obtained for a small number of stars in the 'hook' region of the HR diagram, where a star could be on the main sequence or on the subgiant branch.

The age-metallicity relation (AMR) may be used to study the chemical evolution of the Milky Way. The GCS comprises about $90 \%$ disk, $8 \%$ thick disk and $2 \%$ halo, and we assemble a simulated catalogue with this same mix and apply the same selection criteria and calibrations as were used in the real case. Hence the overall slope in the AMR, with the mean metallicity increasing to younger age, is shown to be real, but an apparent deficiency of metal poor stars at the youngest ages results from the blue cutoff to the sample. This latter point argues against a closed-box model for galactic evolution.

\subsection{Matthias Steinmetz: Radial velocity surveys}

We require radial velocities to provide phase-space information in order to understand how the Galaxy was formed, but by 2000 only about 40000 radial velocities had been determined for stars in the Galaxy, although a million galaxies have measured redshifts. The Geneva-Copenhagen survey provided radial velocities for 20000 stars selected from the Hipparcos catalogue. Two major projects are already under way. The SDSS/SEGUE project aims to obtain radial velocities and metallicities for 250000 stars of magnitude $14.5<g<20$, while RAVE will provide radial velocities and chemical abundances for a million stars, to a limiting magnitude $I=12$, by 2010. SEGUE will observe stars in strips across the Galactic plane in the northern hemisphere, with resolution 2000 over the wavelength range $3800-9100 \AA$. RAVE offers more complete coverage of 15000 square degrees of the southern sky, with a resolution of 7500 over a narrow spectral region, 8460 - $8740 \AA$ A, covering the Ca II triplet. Abundances of many elements will be measured.

SEGUE spectroscopy is supported by accurate and homogeneous multicolour photometry. It will reach at least $100000 \mathrm{pc}$ in dust-free directions and will delineate substructure in the distant halo, the galactic anticentre and the outer disk. The spectra, with $3 \AA$ resolution, will provide radial velocities accurate to $7 \mathrm{~km} / \mathrm{s}$ at $g=18.2$. The expected uncertainties in atmospheric parameters are $150 \mathrm{~K}$ in $\mathrm{T}_{\text {eff }}, 0.3 \mathrm{dex}$ in $[\mathrm{Fe} / \mathrm{H}]$ and $0.5 \mathrm{dex}$ in $\log g$. External checks are being made against stars which have high resolution spectroscopic data, especially in globular and open star clusters. A total of 90000 spectra in 75 fields had been obtained by August 2006; errors of $7 \mathrm{~km} / \mathrm{s}$ were obtained for main sequence turn off stars of the thick disk, with $g-r>0.45$, and $11 \mathrm{~km} / \mathrm{s}$ for metal poor main sequence turn off and blue horizontal branch stars with $g-r<0.45$.

RAVE observes stars with $9<I<12$ with a fixed exposure time of an hour, so the SNR is magnitude dependent. Half the stars observed have errors under $2.0 \mathrm{~km} / \mathrm{s}$ and reobservations indicate that stability is maintained, while external sources of high precision velocities confirm the zero point and scale of the velocities. A total of 131500 stars had been measured by August 2006. Initial results show that errors are generally less than 0.1 dex in $[\mathrm{Fe} / \mathrm{H}], 0.15 \mathrm{dex}$ in $\log g, 2 \%$ in $T_{\text {eff }}$ and $6 \mathrm{~km} / \mathrm{s}$ in $v_{\text {rot }}$. The intensity of the prominent diffuse interstellar band near $8660 \AA$ shows a linear correlation with $\mathrm{E}(B-V)$. A preliminary estimate of the mass of the Galaxy is $1.45 \times 10^{12} \mathrm{M}_{\odot}$.

\subsection{Catherine Turon: astrometric surveys}

Astrometric surveys provide trigonometric parallaxes and proper motions, which together with radial velocities give the full phase space information. Global astrometric surveys, 
covering the whole sky and linked to a reference frame, provide the reference frame for photometric and spectroscopic surveys as well as for small field observations with large telescopes. Homogeneous data sets with clearly defined limits are essential, both in order to control selection effects and to identify the sources of error.

Ground-based surveys, accurate to the mas level, are still limited by atmospheric motions, by the deformation of the telescope under gravity, and by the small field of the telescopes. Attempts to find all the nearest stars used photometric and spectroscopic as well as astrometric data, and some of the findings were contradicted by the accurate Hipparcos parallaxes. This work continues, extended to fainter magnitudes in order to find brown dwarfs, and many more stars have been found near the Sun.

The USNO CCD Astrographic Catalog, UCAC, is an astrometric all-sky catalogue made with the USNO Twin Astrograph. The preliminary UCAC2 contains data for 48 million stars with $R=8-16$ from $-90^{\circ}$ declination to at least $+40^{\circ}$ but lacks stars with $R \leqslant 8$, multiple stars with separations up to $6^{\prime \prime}$ and stars without good proper motions. The final catalogue, UCAC3, will include 60 million stars with positional errors of 15 mas at best and systematic errors smaller than 10 mas, as well as being more complete. The USNO-B1.0 catalogue is based on digital scans of all the major sky surveys from Palomar, $\mathrm{AAO}$ and ESO. It contains magnitudes and star/galaxy estimators for more than a billion objects to $V=21$. The J2000 positions have an accuracy of $0.2^{\prime \prime}$ and relative proper motions are provided. Other important ground-based astrometric surveys are the Carlsberg Meridian Catalogue 14 (CMC14), the Bordeaux Proper Motion Catalogue (PM2000) and NOMAD, a compilation of the best astrometric data. 2MASS and SDSS combine astrometric positions with photometric/spectroscopic data, while USNO-B has been combined with SDSS to give a proper motion catalogue. Major future projects under way or under consideration are URAT, Pan-STARRS and LSST.

Observations in space eliminate the effects of atmospheric seeing and gravitational distortion of the telescope. The instrument has access to the whole sky, so that all observations can be linked. The only survey to date was the Hipparcos mission, which provided proper motions and parallaxes with a median accuracy of about 0.8 mas $/ y r$ and 1 mas respectively, and a link to the International Reference System to 0.6 mas, for 118000 pre-selected stars. Multi-epoch photometry to $0.0001-0.0005$ mag was obtained for them all. The data from the star mappers was used to provide data for 2.5 million stars to $V=11.0$, as the Tycho-2 catalogue.

\subsection{Javier López-Santiago: Young stellar populations in the Solar neighbourhood}

Young stellar populations are best enumerated by stellar X-ray surveys. These enable the age distribution of main sequence stars of low mass to be determined, as the $\mathrm{X}$ ray luminosity of such stars change by 3-4 orders of magnitude even though there is negligible change in optical luminosity. It follows that young stars may be detected at much greater distances than old stars in X-ray flux-limited surveys. The smaller scale heights of younger stars means that old stars will be the dominant population in deep high-latitude stellar X-ray samples, while young stars will dominate in shallow samples. An excess of young stars in the solar neighbourhood was detected by the Einstein-EMSs, EXOSAT and ROSAT missions, excluding the possibility that the stellar birthrate has decreased in the last billion years. The excess stars detected in the EMSS survey were yellow stars, whereas the Chandra Deep Field-North survey shows a lack of F-, G- and K-type stars but many dM-type stars. This difference may be related to the dominance of old disk stars in the CDF-N, while the EMss survey is dominated by young stars.

Two new shallow surveys, the Bright Source Sample (BSS) of the XMM-Newton Bright Serendipitous Survey and the stellar sample of the ROSAT North Ecliptic Pole (NEP) 
Survey, may be used to study stellar populations near the Sun. The BSS provides Xray spectroscopy for 58 stellar sources with count rates of at least $0.01 \mathrm{cnt} / \mathrm{s}$ in the energy range $0.5-4.5 \mathrm{keV}$ at galactic latitude $|b|>20 \mathrm{deg}$. The NEP contains 151 sources detected in the energy range $0.1-2.4 \mathrm{keV}$ in a $9^{\circ} \times 9^{\circ}$ field centred on the NGP. The spectra found in the BSS may be fitted with two components with similar contributions and of temperatures around $0.31 \mathrm{keV}$ and $0.98 \mathrm{keV}$, each with a narrow Gaussian half-width near $0.17 \mathrm{keV}$. Similar spectra are known for the coronae of slowly-rotating solar-type stars in the Pleiades. This suggests that the BSS is dominated by young main sequence stars. Their infrared counterparts lie on the main sequence in the $J H K$ two-colour diagram from 2MASS data. The BSS and NEP samples both comprise mainly main sequence stars of type $\mathrm{G}-\mathrm{M}$.

Comparison of the spectral type distribution of the stars observed in the BSS and NEP surveys with predictions from the X-ray galactic model XCOUNT shows that there is an excess of FGK stars, which would be relatively young, although the X-ray spectra and the position of the stars in the $J H K$ two-colour diagram suggest the stars are of intermediate or old age. A possible solution is that the excess FGK stars are binaries with a yellow primary and an $\mathrm{M}$ dwarf secondary.

\subsection{Daisuke Ishihara: The mid-infrared all sky survey with AKARI}

The first mid-infrared sky survey was completed by IRAS in 1983: almost the entire sky was covered in four broad wavebands centred on 12, 25, 60 and $100 \mu \mathrm{m}$. The later Midcourse Space Experiment (MSX) repeated crowded regions such as the Galactic plane and the Magellanic Clouds, as well as the small area which had not been covered before the helium coolant in IRAS was exhausted. Four broad bands, at 8.28, 12.13, 14.65 and $21.34 \mu \mathrm{m}$, were observed with higher sensitivity and spatial resolution.

The AKARI satellite, formerly known as Astro-F, was launched from Japan into a Sunsynchronous orbit in February; observations began in May 2006. The Ritchey-Chretien telescope has a primary mirror of $685 \mathrm{~mm}$ diameter, cooled to $6 \mathrm{~K}$ by superfluid liquid helium and by mechanical coolers. It will conduct an all-sky survey in the mid infrared using the InfraRed Camera (IRC). This has broad bands centred on $9 \mu \mathrm{m}$ and $18 \mu \mathrm{m}$, with a spatial resolution of 10 arcsec. This relatively high resolution, which is nearly ten times better than that of IRAS, will provide a much better depiction of crowded Milky Way fields and star formation regions. The sensitivity is better than 50 and $120 \mathrm{mJy}$, respectively, nearly ten times better than $I R A S$, so it will be able to reach a T Tauri star at a distance of $100 \mathrm{pc}$. The $A K A R I$-IRC will also undertake imaging and low resolution spectroscopy in pointed mode. Absolute calibration is carried out with a network of 615 bright $\mathrm{K}$ - and M-type giants, which will place the data on the system established by $M S X$, and 249 fainter calibrators, which were set up to calibrate the IRAC camera on the Spitzer satellite. AKARI-IRC observations are thus especially well suited to the study of young stellar objects and debris disk systems, including those in regions of active star formation. The all-sky survey will be important for the study of mass loss in the later stages of stellar evolution.

The Far Infrared surveyor experiment (FIS) on AKARI will also observe the entire sky in four broad bands between 50 and $200 \mu \mathrm{m}$. The sensitivity and angular resolution of FIS also improve on the performance of IRAS by an order of magnitude.

The various instruments are fed from different parts of the focal plane, so that the various wavebands are not observed simultaneously in a specific direction. The two midinfrared bands of the AKARI-IRC are observed in separate fields, and the AKARI-FIS in a third. Each of the IRC detectors covers a field of approximately $10^{\prime} \times 10^{\prime}$ for pointed observations, while the all-sky survey observations use only two of 256 pixels in the 
direction of scan. The virtual pixel size is $4 \times 4$ of the intrinsic pixel size, by virtue of a readout time of a quarter of the dwell time of a point source on a pixel in the scan direction, and by binning four pixels in the cross-scan direction. Each channel of the IRC - the two mid-infrared cameras and the one devoted to the near-infrared - has three broad band filters and two spectroscopic dispersers for low-resolution spectroscopy.

The first AKARI point source catalogues of the IRC and the FIS all-sky surveys are expected to be generally available two years after the end of the survey. A faint source catalogue and a small scale structure catalogue are planned to follow later, as well as image data.

\section{Impact on Galactic astronomy}

Reported by Coryn A.L. Bailer-Jones

\subsection{Joss Bland-Hawthorn: Galactic history - formation and evolution}

The primary motivation for large surveys, both past/ongoing (Hipparcos, SDSS, SEGUE, RAVE) and future (pan-STARRS, Gaia, WFMOS), is, arguably, exploring the formation and evolution of galaxies. This topic will dominate observational cosmology and galactic studies for decades to come, mostly because it is difficult to define a unique model for galaxy formation (assuming one exists) which fits the diversity of observations. It is not sufficient to simply identify the building blocks of galaxies at high redshift and to demonstrate that numerical simulations can explain their properties and those of galaxies at later ages. We must also construct a detailed picture which self-consistently includes the underlying physics.

Observations indicate quite conclusively that galaxies form through the gradual buildup of dark matter and baryons. Both the Milky Way and M 31 show (sub)structure in most of their components. A major development over the past decade started with the discovery of a dwarf spheroidal galaxy in Sagittarius that was seen to be falling into the Galactic disk, plus a tidally disrupted stream associated with this. Numerous other streams have since been discovered in the halo of the Milkey Way. (Some argue that most or all of these Milkey Way streams are either associated with the Sgr dSph or are part of the outer disk.) Hipparcos found evidence for substructure in the thin disk: the distinct ages of these clumps suggest that they are dynamical in origin rather than a result of patchy star formation. A large tidal stream has also been discovered associated with M31. Indeed, the (metal poor) halo of M31 is now believed to extend to at least $140 \mathrm{kpc}$, some $20^{\circ}$ on the sky. M33, a satellite to M31, has been found to have a halo with a similar metallicity to those of both M 31 and the Milkey Way.

It can be argued that establishing a theory of galaxy formation is primarily about understanding the processes which formed disks in the early universe. The thick disk of the Milkey Way is about 10-12 Gyr old and, compared to the thin disk, is dynamically 'hotter' and more metal poor (also with different alpha element abundances, as discussed below), possibly with a distinct abundance gradient and longer radial scale length. One interpretation is that the thick disk was formed through dynamical heating from the merger of another galaxy. Other scenarios for forming the thick disk include dissipational collapse of gas or that it is the dispersed remnants of unbound star clusters in the early universe. Recent observations suggest that thick disks are common in galaxies, so we are probably not dealing with a rare event for which an ad hoc explanation suffices.

The Local Group is a relatively low density cluster. As there are indications that galaxy formation depends on environment, future surveys must extend out to the nearest dense clusters, such as Virgo. This will be possible with JWST, but optical and UV observations 
are also necessary to determine metallicities, perhaps from ground-based $30-40 \mathrm{~m}$ class telescopes. Of particular interest is the origin of galactic bulges and halos. For example, while the Milkey Way and M 31 are comparable in mass, M 31 has a much more prominent bulge. What caused this? To answer this, high spatial resolution infrared surveys are essential. Ancient stars are important for understanding old halo populations: these very low metallicity stars are probably some of the first stars to have formed in the universe. Their exact abundances and distributions will tell us about the earliest phases of star formation in proto-galaxies.

Extragalactic surveys provide support for the $\Lambda$ CDM paradigm for galaxy formation, and computer simulations yield structures which look a lot like real galaxies and galaxy clusters. But there are problems. For example, these models predict higher densities of dark matter in the cores of galaxies than are inferred from observations. Self-consistent models of the Galactic bulge also suggest that baryons dominate the total mass within the Solar Circle, in contradiction with CDM simulations. To improve our picture of galaxy formation, future surveys will need to combine information on both the kinematic phase space (positions and velocities) and the chemical phase space (abundances of various elements with different product paths).

\subsection{Sofia Feltzing: Abundance structure of the Galactic disk}

Stars in the solar neighbourhood can be tagged to different stellar populations (e.g., thin disk, thick disk, halo) based on their kinematics, abundances and ages. Stars in the Galactic disk with distinct kinematical signatures have distinctly different elemental abundances. Specifically, for stars at a given $[\mathrm{Fe} / \mathrm{H}]$, those with thick disk kinematics have higher abundances of the alpha elements (O, Ne, Mg, Si, S, A, Ca, synthesized and released predominantly by Type II, or core collapse, supernovae) than do stars with thin disk kinematics. Oxygen in particular is important because it is produced almost exclusively in SN II, the progenitors of which are massive stars with lifetimes of tens to one hundred million years. Other elements are produced both by SN Ia and SN II. Hence the $[\mathrm{O} / \mathrm{Fe}]$ abundance can be used a clock to trace the relative contributions of SN Ia and SN II. The trends in the $[\mathrm{O} / \mathrm{Fe}]$ vs. $[\mathrm{Fe} / \mathrm{H}]$ plot (Fig. 1) can be interpreted to indicate that at early times (clocked by $[\mathrm{Fe} / \mathrm{H}] \simeq-0.5$ ), the thick disk (filled circles) underwent a period of intense star formation. Only SN II would have been operational at this time, and so the $[\mathrm{O} / \mathrm{Fe}]$ ratio remained constant. Later, SN Ia start to contribute, thus increasing the ratio of $\mathrm{Fe}$ compared to $\mathrm{O}$, and so $[\mathrm{O} / \mathrm{Fe}]$ decreases as $[\mathrm{Fe} / \mathrm{H}]$ increases. The plot for the thin disk stars is slightly different and implies a lower star formation rate with more equal contributions from SN Ia and SN II and therefore a smoother trend across the whole plot.

Analyses of other elements reveal the following. [C/Fe] shows similar trends amongst thin and thick disk stars. (This is based on carbon abundances derived from a forbidden transition and so is robust to departures from LTE in the stellar atmosphere.) This implies that the sources which produce carbon operated on the same timescale as those which produced iron (which is SN Ia). If we could nail down the production of carbon we could infer the evolution of the SN Ia rate. Unfortunately, the formation of carbon is not yet well understood, with no unique observational picture emerging. A survey of 95 kinematically-selected dwarfs shows that the stars with thin disk kinematics have enhanced levels of manganese relative to oxygen $([\mathrm{Mn} / \mathrm{O}])$ compared to the stars with thick disk kinematics. Furthermore, for thick disk stars [Mn/Fe] steadily increases with increasing $[\mathrm{Fe} / \mathrm{H}]$, whereas for thin disk stars it is flat below $[\mathrm{Fe} / \mathrm{H}]=0$ and increases above that value. Plotting instead against $[\mathrm{O} / \mathrm{H}]$ (which makes more sense as oxygen is only produced in SN II) we see that for thick disk (and halo) stars, $[\mathrm{Mn} / \mathrm{O}]$ is flat below 


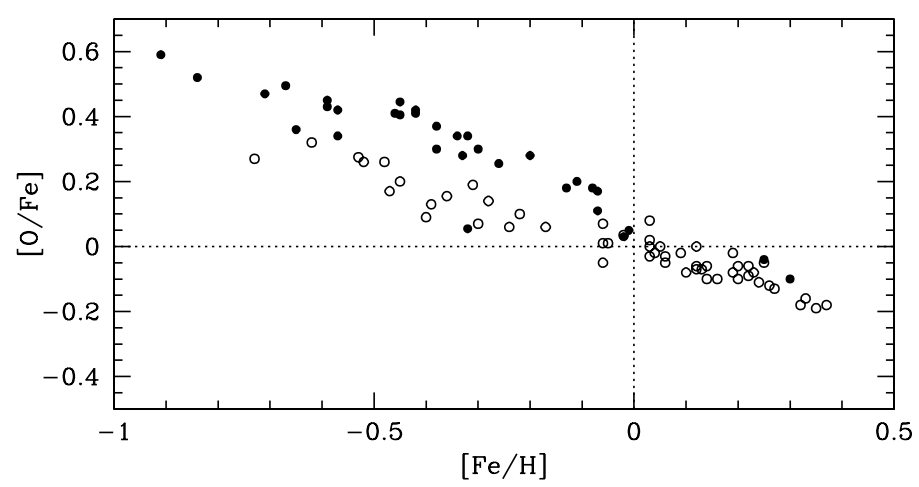

Figure 1. $[\mathrm{O} / \mathrm{Fe}]$ vs. $[\mathrm{Fe} / \mathrm{H}]$ for two samples of stars. • denote stars that have kinematics typical of the thick disk whilst o are stars with kinematics typical of the thin disk. From Feltzing (2006) in the proceedings of the JD13 published in MemSAI.

$[\mathrm{O} / \mathrm{H}]=-0.5$. This indicates a common or synchronized $\mathrm{Mn}$ and $\mathrm{O}$ production. Above $[\mathrm{O} / \mathrm{H}]=-0.5$ the $[\mathrm{Mn} / \mathrm{O}]$ abundance rises, which is interpreted as metallicity-dependent yields in SN II.

Upcoming surveys will make a significant impact in this field. Most spectroscopic abundance surveys have been confined to the solar neighbourhood. Deep photometric surveys will provide targets at larger distances, in particular dwarfs (which are easier to model) which can then be observed spectroscopically on large telescopes to measure individual elemental abundances. Geometric distances (i.e., parallaxes) are required to then map the abundance structure of the Galactic disk, and accurate proper motions allow us to extend abundance - velocity analyses to larger volumes. Parallaxes are also necessary to determine the evolutionary phase or surface gravity (which cannot always be deduced unambiguously from line broadening) which is vital for determining the elemental abundances. Finally, we also need age estimates. These are notoriously difficult and/or inaccurate to estimate for individual field stars, and can only be obtained with any accuracy for MS turn-off stars or for young stars where we believe chromospheric activity proxies. While thick disk stars appear, on average, to be older than thin disk stars, there is still a debate concerning how well separated their age distributions are. Larger samples will help.

\subsection{Amina Helmi: Dwarf spheroidal satellites of the Galaxy}

Most of the satellite galaxies of the Milky Way are very low luminosity systems, the dwarf spheroidals (dSph). While they show a large variety of star formation and chemical evolution histories, all contain a population of old, metal-poor stars (with metallicities similar to stars in the Galactic halo). Because the metallicity of a galaxy increases with time (due to enrichment by successive generations of stars of the interstellar medium, ISM), the metal poor stars are presumably some of the earliest to have formed in the universe. The red giant branch stars in these dSphs are believed to contain in their atmospheres a representative sample (unpolluted by dredge-up) of the chemical elements of the ISM from which they formed.

The DART team has carried out a spectroscopic study of the RGB stars in four dSphs: Sculptor, Fornax, Sextans and Carina. Their CM diagram for Sculptor is shown in Fig. 2. The metallicites are determined from the EW of the individual Ca II triplet lines and radial velocities via cross correlation. Members are then selected via radial 


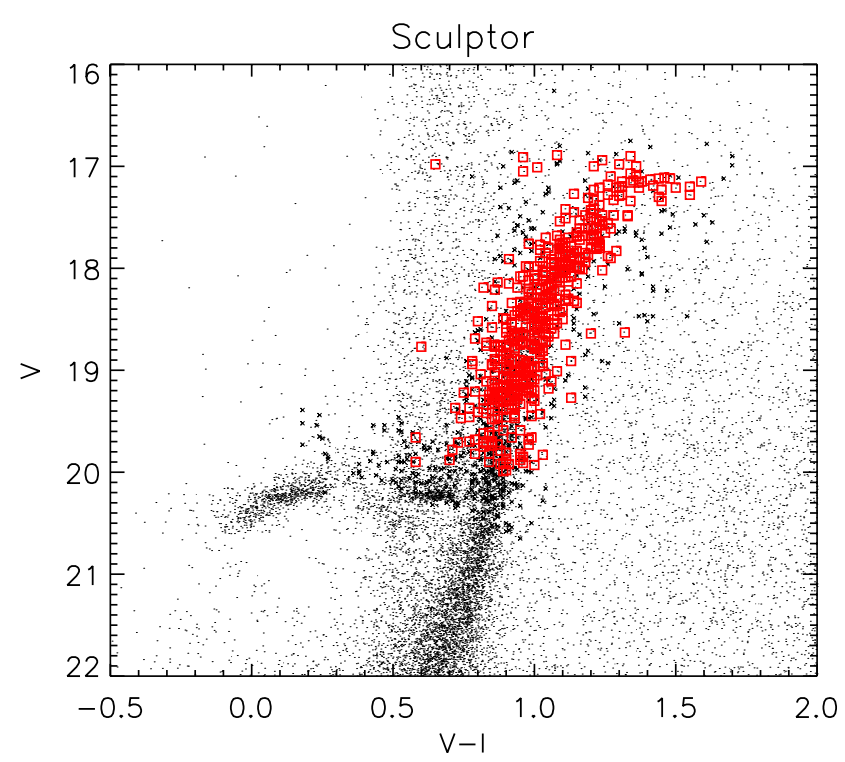

Figure 2. Colour-magnitude diagram of Sculptor. Light squares denote kinematic members and the dark asterisks probable foreground stars, as identified spectroscopically. From Helmi (2006) in the proceedings of the JD13 published in MemSAI.

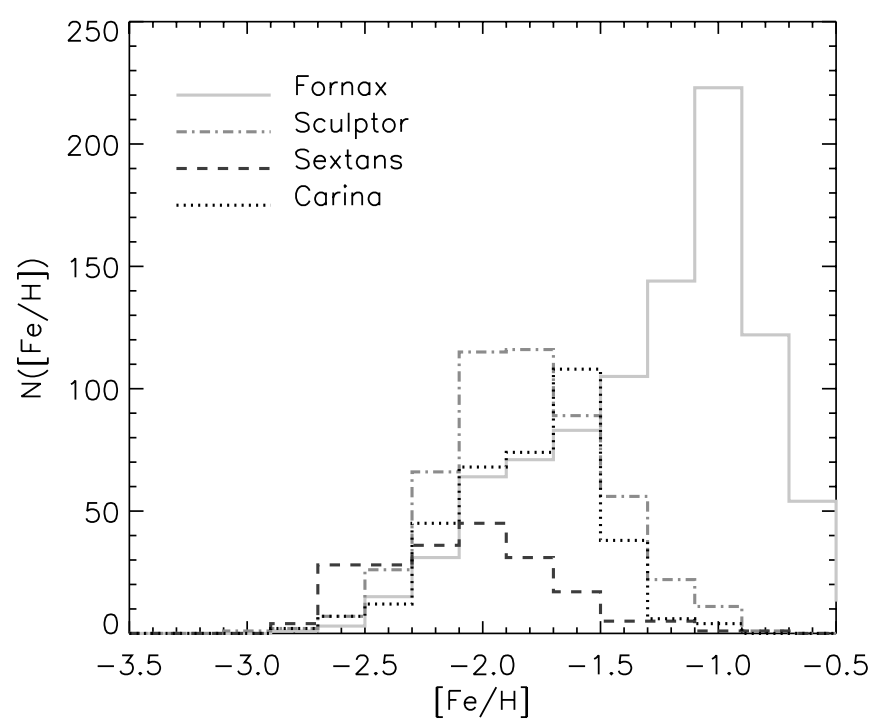

Figure 3. The metallicity distribution for the stars in four dSph. There is an evident lack of objects more metal-poor than $[\mathrm{Fe} / \mathrm{H}]<-3$ dex. From Helmi $(2006)$ in the proceedings of the JD13 published in MemSAI.

velocity and spectral type. The frequency distribution over metallicity for the RGB stars differ between the four dSph (Fig. 3), yet all have one thing in common: the lack of stars with $[\mathrm{Fe} / \mathrm{H}]<-3.0$. Using a closed-box model of chemical evolution with initial enrichment shows that the gas in all four of the dwarf galaxies must have been enriched 
to $[\mathrm{Fe} / \mathrm{H}] \simeq-3.0$ prior to the earliest epoch of star formation which led to the present observed population. The fact that this is the same in four otherwise very different $\mathrm{dSph}$ galaxies implies that the pre-stellar gas was uniformly enriched over a large volume $\left(\sim 1 \mathrm{Mpc}^{3}\right.$, the volume of the Local Group).

In contrast, the low metallicity tail of the Galactic halo extends well below $[\mathrm{Fe} / \mathrm{H}]=-3.0$. Models indicate the Milky Way halo must have had a very low initial enrichment of $[\mathrm{Fe} / \mathrm{H}]=-4.5$ or less, even consistent with no initial enrichment $([\mathrm{Fe} / \mathrm{H}]=-\infty)$.

One idea for the hierarchical build-up of galaxies, and our Galaxy in particular, is that they formed via the accretion of smaller satellites. What is the relation between these putative satellites and the dSph galaxies? To answer this we must compare the metal poor (i.e., old) Galactic halo stars not with the present-day properties of the dSph galaxies but rather with their properties at the time they would have been accreted. The building blocks which accreted onto the Galaxy many billions of years ago had little time to evolve as independent entities, unlike the dSph galaxies we see now (which, of course, were not accreted). Therefore, to make the comparison with the metal poor halo stars, we must focus on the metal poor stars (only) in the dSph galaxies, as these too presumably correspond to their first stellar populations. The conclusion: The fact that the Galactic halo has stars of significantly lower metallicity indicates that it cannot have formed via accretion of the progenitors of the present-day dSph galaxies. Statistical tests (bootstrap and Kolmogorov-Smirov) suggest that this conclusion is not due to the relatively small number of stars in the comparison.

A possible explanation of this conclusion is that whereas the Galaxy formed from a high- $\sigma$ density fluctuation in the early universe (in the $\Lambda$ CDM paradigm), the lower mass $\mathrm{dSph}$ galaxies formed from the low- $\sigma$ fluctuations. The latter are predicted to collapse (on average) much later. Before the dSph galaxies formed (not before $12 \mathrm{Gyr}$ and so after reionization) the intergalactic medium could have been pre-enriched. Alternatively the initial mass function could have differed in the early dSph and in the Galactic building blocks, thus altering their chemical evolution.

\section{Survey data: gains, limitations and challenges Reported by Sunetra Giridhar}

The new and ongoing surveys are providing a large volume of data comprised of positions, kinematics, multi-band photometry and in some cases spectroscopy at moderate resolutions, reaching out farther in distances, with extended wavelength coverage and with better precision than before. The stellar parameters derived from such extended data sets have the potential of making a large impact on our understanding of the structure and formation of our Galaxy, as well as that of the Local Group, by providing observational input as well as constraints on the models of galaxy evolution. However, careful analyses of these datasets from various surveys and attempts at normalization/standardization of these datasets by experts have also exposed certain gray areas about which the users are being cautioned. A versatile approach based on combining carefully chosen multi-band photometry with spectroscopy could lead to more precise stellar parameters for objects belonging to all parts of the HR diagram. Due to their objectivity and capacity of handling large datasets, automated methods of classifications and parameterization such as those based on Neural Network are extremely important and would become a necessity rather than a choice for the datasets from future surveys. The population synthesis approach is known to be useful for the analysis of large datasets. The consolidated stellar information from these datasets, such as evolutionary stages, kinematics and metallicity and $3 \mathrm{D}$ extinction distribution, would be used to test global scenarios of formation and 
evolution of the Galaxy, including kinematical, dynamical and chemical evolutionary constraints. However, developing accurate and efficient methods for data handling would be a challenging task and should be given high priority if we want to get maximum benefit from the present and future surveys.

\subsection{Ian S. Glass: Classic and new photometric systems}

Many large surveys are based on a new system of color bands. These new multi-band surveys offer some sort of classification and help in identifying unusual objects. A good example is the filters used in search of brown dwarfs of type $\mathrm{T}$ that show hot star colors in $J-H$ vs. $H-K$ two-color diagrams due to extraordinary molecular features in their spectra, e.g., $\mathrm{H}_{2} \mathrm{O}, \mathrm{CH}_{4}$ and $\mathrm{H}_{2}$ bands. By adding $Y(1.02 \mu \mathrm{m})$ and $Z(0.89 \mu \mathrm{m})$ to the conventional $J H K$ system, these cool dwarfs can be better discriminated in the $Y-J v s$. $J-H$ diagram. However, use of these uncommon filters also requires new observations of standard stars whose magnitudes are within the range of the new system, new tables of intrinsic colors, new models, new corrections of interstellar absorption, etc., if high precision is to be achieved. It should also be remembered that transformations and color equations are valid only for smooth spectra without deep absorptions and emission lines. Hence the colors of late type stars or QSOs having strong and variable emission lines cannot be transformed.

Some of the new frontiers such as planetary transits, studies of star-spots and high modes of stellar oscillations require very high precision photometry. A precision of one part in $10^{4}$ can be achieved only with very stable instrument response and seeing conditions, and only when the comparison star is present in the same CCD frame.

In studies of the galactic disk, the extinction $\mathrm{A}(\lambda)$ may vary significantly across the pass-bands of a broadband filter. The problem becomes more acute in IR where the functional form of $\mathrm{A}(\lambda)$ beyond $2.5 \mu \mathrm{m}$ changes from a simple power law and appears to flatten out until the region of the $\mathrm{SiO}$ band beyond $8 \mu \mathrm{m}$. Better determination of $\mathrm{A}(\lambda)$ can be found in Nishiyama et al. (2006).

\subsection{Richard O. Gray: Parameterization of stars}

The Strömgren uvby $\beta$ system has been successfully used in parameterization of stars over the spectral type range B through G. A photometric system comprising 5 broad and 14 medium pass-bands, as envisaged for Gaia (but later replaced by low resolution spectra), had versatility to characterize stars over much of the HR diagram as explained in Jordi et al. (2006). The proposed low-resolution option for Gaia may offer opportunities to overcome the problems inherent to photometry. The photometric strategies involving construction of a spectral energy distribution (SED) (or at-least sizable part of it), by converting available magnitudes for a star into fluxes and comparing them with those predicted by model atmospheres, may be adequate for certain stellar parameters such as the temperatures. The method is not sensitive to other parameters like metallicity. Better photometric strategies of stellar parameterization employ carefully calibrated photometric indices, e.g., the $\mathrm{T}_{\text {eff }}$ calibration of Alonso et al. (1996), the reddening correction of Olsen (1988), the $[\mathrm{Fe} / \mathrm{H}]$ calibration of Nordström et al. (2004). But all these calibrations have a strict limit of validity and hence cannot be applied to surveys dealing with stars with a vast range in stellar parameters. The photometric systems do suffer from local and global degeneracies. An illustrative example is the global degeneracy between $\mathrm{T}_{\text {eff }}$ and $\mathrm{A}_{v}$, made more difficult to handle by the fact that the extinction law varies from place to place in the Galaxy.

A better method based on artificial neural network in being explored by Bailer-Jones and his colleagues. This system maps the observation space into parameter space and 
provides 'super' calibration. However, such a system needs to be trained initially with photometric indices derived from stellar spectra. The difficulty with this approach is that synthetic spectra (in some parts of the HR diagram) do not adequately reproduce actual spectra. Since we train the neural network using the data of normal stars, the system will have difficulty in recognizing truly unusual and astrophysically important objects, which would certainly be present in new surveys covering hundreds of million objects.

Since Gaia spacecraft design has substituted the multi-color photometric system with low resolution prism spectra, the SEDs generated with these flux calibrated spectra may be used to derive stellar parameters. But instead of comparing these fluxes to those obtained from models directly (which would be equivalent to doing an unweighted fit to the data), Bailer-Jones attempted to optimally sample the SED using filter parameters as free parameters that could be determined by optimizing how well the filter system discriminates between stellar types and avoids degeneracies. The resulting filter system had broad overlapping bands, and these broad bands would be compatible with the low-resolution SEDs to be obtained by Gaia. Using this finding one may contemplate designing optimal pass-bands or weighting for different spectral types. The analysis pipeline may first determine a rough stellar type and then apply the pass-bands optimized for that particular stellar type to determine physical parameters. This approach may be able to overcome many degeneracies of traditional fixed photometric systems.

The strategy of adding spectra to photometry has been adopted in the Nearby Stars (NStars) program wherein all dwarfs and giants earlier than M0 within $40 \mathrm{pc}$ of the Sun, as determined by the Hipparcos mission, were characterized. Here the Strömgren uvby system is augmented with ultraviolet fluxes for the hotter stars and with JohnsonCousins $R I$ photometry and medium resolution spectra in the blue-violet region. The stellar parameters are determined by simultaneously fitting synthetic spectra and fluxes to the blue-violet spectra and fluxes from photometric sources. This strategy resulted in well-constrained fits over a wide range in stellar parameters and the parameters could be determined precisely with fewer spurious solutions. The blue-violet spectral region used in NStars would not be suitable for surveys covering objects with large extinction in the Galactic plane. Gaia and RAVE have chosen the $840-880 \mathrm{~nm}$ region due to its absence of telluric lines and since it contains important lines like the Ca II infrared triplet (sensitive to $\mathrm{T}_{\text {eff }}$ and gravity as well as to chromospheric activity), diffuse interstellar lines to estimate extinction, lines of $\alpha$-elements and Paschen series lines of hydrogen; but this spectral region is not really suitable for parameterization of early-type stars. Secondly, even for F-G type stars lines in $840-880 \mathrm{~nm}$ are a slowly varying function of temperature, and a metal-poor early-G star looks very similar to a mid-F type star. This degeneracy may lead to a poor determination of temperature and/or metallicity. A well designed photometric system, combined with spectroscopy, and in a carefully chosen region of the spectrum, is an ideal approach, and one that opens the survey to measuring much more than basic physical parameters since the spectra and photometry work in complementary ways, where one makes up for the weakness of the other.

\subsection{Sunetra Giridhar: Automated classification and stellar parameterization}

Many large telescopes are now equipped with multi-object spectrometers enabling coverage of a large number of objects per frame for stellar systems like globular clusters. On-going and future surveys and space missions would collect a large number of spectra for stars belonging to different components of our Galaxy. Such a large volume of data can be handled only with automatic procedures, which would also have the advantage of being objective and providing a homogeneous data set most suited for Galactic structure and evolutionary studies. Another outcome would be the detection of stellar variability 
and finding peculiar objects. Although Minimum Distance Method (MDM) and Gaussian Probability Method (GPM) have been popular, automated spectral classification using Principal Component Analysis (PCA) and Artificial Neural Network (ANN), would have wider applications now and in the future.

PCA is a method of representing a set of N-dimensional data by means of their projection onto a set of optimally defined axes. Since these axes (Principal components) form an orthogonal set, a linear transformation of the data is achieved. Components that represent large variance are important, while those that represent least variance can be ignored and the data set can be replaced by significant components alone, thereby resulting in a reduction of the data size. These compressed data sets are used as input for neural networks. Bailer-Jones et al.(1998) had demonstrated that precise calibration could be done using these compressed spectra and that the optimal compression also results in noise removal. Neural Network is a computational method which can provide non-linear parameterized mapping between an input vector (a spectrum for example) and one or more outputs like SpT and LC, or $\mathrm{T}_{\text {eff }}, \log g$ and $[\mathrm{M} / \mathrm{H}]$. The method is generally supervised; this means that for the network to give the required input-output mapping, it must be trained with the help of representative data patterns. These are stellar spectra for which classification or stellar parameters are well determined. The training proceeds by optimizing the network parameters (weights) to give minimum classification error. Once the network is trained and the weights are fixed, the network can be used to produce output $\mathrm{SpT}$ and $\mathrm{LC}$, or $\mathrm{T}_{\text {eff }}, \log g$ and $[\mathrm{M} / \mathrm{H}]$ for an unclassified spectrum. A summary of the application of ANN can be found in Bailer-Jones (2002).

At IAAP we have made a modest effort to use ANN for parameterization of a sample of stars in the temperature range 4500 to $8000 \mathrm{~K}$ using medium resolution spectra ( $R \simeq 1000$ ) obtained with the $2.3 \mathrm{~m}$ Vainu Bappu Telescope at VBO, Kavalur, India. The spectra have a wavelength coverage of $3800-6000 \AA$. The stars from the list of Allende et al. (1999) and Snider et al. (2001) were included to develop a library of stars with known temperatures, gravities and metallicities. These spectra were used for training and testing the network. The preliminary results based on 680:11:3 architecture gave stellar parameters with RMS errors of $200 \mathrm{~K}$ for $\mathrm{T}_{\text {eff }}, 0.3$ dex for $[\mathrm{Fe} / \mathrm{H}]$ and $0.4 \operatorname{dex}$ for $\log g$.

It is very important to envisage an approach that would give quick, reliable spectral classifications (or stellar parameters) for stars falling in all regions of the HR diagram. A single ANN architecture may not give the same desired accuracy over the full range of spectral types and luminosity classes. A pilot program, using the photometric inputs in visual and UV, and using special photometric indices measuring the strengths of molecular bands for late-type stars, could serve as preprocessor and help in identifying a set of specialist networks which would lead to classification of the desired accuracy. A specialist system also needs to be evolved for A-type stars to give quick identification of chemically peculiar, magnetic or emission line stars. A special network needs to be developed for objects displaying complex spectra such as symbiotic stars, novae and supernovae. Here the network must be trained on flux calibrated spectra, and it must use emission line strength as well as shape and structure of the continuum (a composite for symbiotic stars and novae) for classification purposes.

\subsection{Annie C. Robin: Data sets and population synthesis}

The population synthesis approach aims at assembling current scenarios of galaxy formation and evolution, theories of stellar formation and evolution, models of stellar atmospheres and dynamical constraints, in order to make a consistent picture explaining currently available observations of different types (photometry, astrometry, spectroscopy) at different wavelengths. The Galactic model generally describes a smooth Galaxy, while 
inhomogeneities are observed in the disk as well as in the halo. It is intended to produce a useful tool to compute the probable stellar content of large data sets and therefore to test the usefulness of such data to answer a given question in relation to Galactic structure and evolution.

The originality of the Besançon population synthesis models lies in its dynamical selfconsistency. The Boltzmann equation allows the scale height of an isothermal and relaxed population to be constrained by its velocity dispersion and the Galactic potential. The use of this dynamical constraint avoids a set of free parameters quite difficult to determine: the scale height of the thin disk at different ages. It gives the model an improved physical credibility. The main scheme of the model is to reproduce the stellar content of the Galaxy, using some physical assumptions and a scenario of formation and evolution. We essentially assume that stars belong to four main populations: the thin disk, the thick disk, the stellar halo (or spheroid), and the outer bulge. The modeling of each population is based on a set of evolutionary tracks, assumptions on density distributions, constrained either by dynamical considerations or by empirical data, and guided by a scenario of formation and evolution.

The thick disk formation scenario has been studied using photometric and astrometric star counts in many directions, which also provided its velocity ellipsoid, local density, scale height, and mean metallicity. These physical constraints led to a demonstration that the probable origin of the thick disk is an accretion event early in the history of the Galaxy. The 3D extinction model has been inferred from star counts and color distribution from the 2MASS survey by Marshall et al. (2006). It furnishes an accurate description of the large scale structure of the disk of dust. It shows a scale height of $98 \pm 21 \mathrm{pc}$. A big hole almost free of dust is identified around the Galactic center with a radius of $\sim 3.2 \pm 0.5 \mathrm{kpc}$, but it also contains an elongated feature which resembles a dust lane with an inclination with regard to the Sun-Galactic center direction of $\sim 32^{\circ} \pm 10^{\circ}$. This feature may trace the dust falling into the center along the stellar bar. This $3 \mathrm{D}$ extinction map can be used to make detailed prediction of the star density and luminosity in the Galactic plane.

The microlensing survey also provides very important constraints. The microlensing optical depth, that is the instantaneous number of ongoing microlensing events per source star, is a key measurable for these surveys and provides an important constraint on the bulge surface mass density. Its dependency upon direction provides, in principle, a unique and powerful probe of the three-dimensional geometry of the bulge stellar mass distribution.

The data sets from different surveys need to be combined to enrich as well as refine the global scenario of formation and evolution of the Galaxy. An accurate and efficient method of data fitting for such large data sets is a primary requirement.

\section{Future strategies}

\section{Reported by Coryn A.L. Bailer-Jones}

\subsection{Coryn A.L. Bailer-Jones: Prospects for Gaia and other space-based surveys}

Space-based surveys are essential for various types of observations and naturally complement ground-based surveys. A space platform is required for the UV, mid- and farinfrared and X-ray, and provides a lower background in almost all wavebands. It permits diffraction limited observations and allows us to overcome temporal and spatial variations of the atmosphere's refractivity, which limit the precision of wide-field astrometry to about 1 mas. 
Several upcoming missions with a significant Galactic component take advantage of this environment. Herschel is a far infrared and sub-mm ESA observatory due for launch in 2008. It comprises several imaging and spectroscopic instruments operating between 60 and $670 \mu \mathrm{m}$. Its key science objective is the formation of stars and galaxies via deep imaging surveys. It will investigate the formation and evolution of galaxy bulges and elliptical galaxies during the first third of the present age of the universe, determining how the galaxy luminosity function and star formation rate has evolved with time. JWST, in contrast, will observe between 0.6 and $27 \mu \mathrm{m}$ and is optimized for performing deep 'pencil beam' surveys. It has four instruments operating over different wavelength ranges, three of which have spectroscopic modes with resolving powers between 100 and 7000 . One of the main science objectives of JWST is to study the early universe, in particular the epoch of the first stars and the formation of the first galaxies. JWST's current launch date is 2013. SIM PlanetQuest is a pointed astrometric mission. Several key programmes on Galactic structure have been approved, including calibration of the stellar MassLuminosity relation, measuring the distances and ages of globular clusters and measuring the Galactic potential via stellar proper motions. At the time of writing (August 2006), SIM faces a very uncertain future due to the shifted priorities of NASA. A launch cannot be expected (if at all) before 2015 .

Gaia is an all-sky astrometric and photometric survey due for launch in 2011. It will measure accurate parallaxes and proper motions for everything brighter than $G=20$ $\left(V=20-22\right.$; ca. $10^{9}$ stars $)$. Its primary objective is to study the composition, origin and evolution of our Galaxy from the 3D structure, 3D velocities, abundances and ages of its stars. Gaia will achieve an astrometric accuracy of $12-25 \mu$ as at $G=15$ (providing a distance accuracy of $1-2 \%$ at $1 \mathrm{kpc}$ ) and $100-300 \mu$ as at $G=20$. (These numbers are also the approximate parallax accuracy in $\mu$ as and the proper motion accuracy in $\mu$ as/year.) It will measure distances to better than $1 \%$ for about 11 million stars, compared to about 200 now.

One of the main contributions of Gaia will be mapping structure across the Galaxy in position, velocity and chemical abundance. This includes small scale structure which could be the remnants of past mergers, which are predicted (and to some extent already observed) to be a major mechanism of galaxy formation. Gaia will also map the dark matter in our Galaxy via two derivatives of its survey. First, from the 3D stellar kinematics we can map the total gravitational potential (dark and bright). Second, using its parallaxes and photometry we can make a detailed and accurate measurement of the stellar luminosity function over a large volume. This is converted into a mass function with an $M-L$ relation which itself will be determined to higher accuracy than is presently possible (and over a wide mass range) using Gaia binaries. Subtracting the stellar mass distribution from the total mass distribution yields the dark matter distribution. Gaia will address many other areas of astrophysics, including calibrating the extragalactic distance ladder, identifying Near-Earth Objects and discovery and characterization of exoplanetary systems and their host stars. In the area of stellar astrophysics, accurate stellar luminosities for stars in open and globular clusters can be derived from their parallaxes and line-of-sight extinctions (determined from the onboard spectrophotometry). From this we can derive the bulk Helium abundance and investigate phenomena such as convective overshooting and diffusion.

The data processing for Gaia is complex and challenging, unlike anything yet undertaken in astronomy. Gaia continuously sweeps the sky for five years. The basic data product is several image strips more than two million degrees long (7000 great circle scans), with each of the one billion objects appearing about 100 times in each strip. The tiny relative displacements within the strip allow us to determine the parallax (distance) 
and proper motions. As Gaia is self-calibrating (i.e., the science data are also the calibration data), the data reduction involves a simultaneous iterative solution of hundreds of millions of source and calibration parameters. On top of this are many other tasks: treatment of binary stars; General Relativistic effects; photometric and CCD calibration; spectroscopic extraction; source classification; variability detection; just to mention a few.

The next significant advance in understanding the formation, structure and evolution of galaxies will come about from three lines of pursuit. The first is detailed astrometric and chemical abundance surveys of our own Galaxy. This is addressed primarily by Gaia, but also by SIM and Jasmine if they fly. While our Galaxy retains fossils of its evolution, these will only ever tell us part of the story, and then only for one galaxy. The second line of pursuit, therefore, is the observation of galaxies at different stages of their life, i.e., at a range of redshifts. Several ground- and space-based surveys are already addressing this, but the next generation of satellites, in particular JWST and Herschel, will focus much more on the earliest epoch of galaxy formation (and star formation) in the high-redshift universe. Together, these two lines of pursuit will significantly advance our understanding of galaxy formation, dark matter, chemical evolution and stellar structure and evolution (to know galaxies we must know stars). The third line is the development of powerful models and data analysis tools. These are essential for processing and then exploiting the Gaia data, but will also be required to draw together knowledge obtained from our 'near-field' cosmological studies (our Galaxy) with that from high-redshift galaxies. Effort must be invested into developing models and data analysis techniques with as much zeal as the instrumentation and space platforms.

\subsection{Nick Kaiser: Pan-STARRS}

Pan-STARRS is a deep imaging survey of the sky. It is split into two phases. The first, PS1, comprises a single $1.8 \mathrm{~m}$ telescope with a seven square degree field-of-view imaging in five filters: $g, r, i, z, y$. Its major survey mode is to observe the $3 \pi$ steradian north of $\delta=-30^{\circ}$ (31000 square degrees) twice every month. The PS1 science observations will start in March 2007 and take 3.5 years. At the end of this, every point in the survey area will have been observed 12 times, and the predicted $5 \sigma$ point source sensitivity is $24.6 \mathrm{mag}$ in $g, 24.0$ in $i$ and 21.5 in $y$. This is 1.3 mag deeper than SDSS (in $g$ ) and covers almost four times the area on the sky.

PS1 has numerous science goals, including the identification of potentially hazardous Near-Earth Objects, constraints on dark energy and dark matter (e.g. via weak lensing), detection of microlensing events and stellar/exo-planet transits, the stellar and substellar mass function and, of particular relevance to this JD, Galactic structure. PS1 will contribute a deep photometric and astrometric survey of $3 / 4$ of the sky. The final catalogue should achieve an absolute photometric accuracy of about $0.01 \mathrm{mag}$ and a relative astrometric accuracy of 10 mas (absolute 100 mas) for source-noise limited observations. Given 3.5 years of observations, relative proper motions precise to a few mas/yr (and parallaxes to a few mas) should be possible, limited by the Earth's atmosphere. Although not as accurate as Gaia, it goes much deeper. This catalogue will permit numerous studies in Galactic structure, such as the search for streams in/around the Milky Way and local group galaxies, luminosity function, identification of bound structures, etc.

PS1 is also intended as a test-bed for the larger PS4 survey, which will have four times the collecting area. This will follow once PS1 is complete and address similar science goals, but to fainter magnitudes and higher precisions. 


\section{A topic-ordered list of poster papers}

This ordering of the posters presented at the Joint Discussion 13, Exploiting Large Surveys for Galactic Astronomy, follows that of Birgitta Nordström, with due thanks.

Most of the actual posters can be found, with their abstracts, at the full, electronic version of the proceedings in Memorie della Società Astronomica Italiana, volume 77 , n.4 at <http://sait.oat.ts.astro.it/>

Their original abstracts are also available at the IAU XXVI ${ }^{\text {th }}$ GA site, <http://www. astronomy2006. com/list-of-registered-abstracts.php?event=jd13>.

\subsection{New and recent surveys}

Radio observations of the HII region complex RCW 95

U. Barres de Almeida, Z. Abraham and A. Roman-Lopes

The Digitized First Byurakan Survey (DFBS): a unique database for proper motion,variability studies, and object classification

A.M. Mickaelian, K.S. Gigoyan, R. Nesci, C. Rossi

A 2dF Survey for Omega Centauri Members at and beyond the Tidal Radius G.S Da Costa, M.G Coleman

Proper motion sky survey of 2.7 million stars with the Bordeaux automated CCD meridian circle

C. Ducourant, J.F. Le Campion, M. Rapaport and 9 co-authors

Survey of open star cluster. Optical monitoring and photometry

A.S. Hojaev

Near infrared survey of the nuclear region of the Milky Way

U.C. Joshi, S. Ganesh, K.S. Baliyan, I.S. Glass and T. Nagata

The search for Post-AGB stars with dusty discs

T. Lloyd Evans, A.M. Smith, J. McCombie, P.J. Sarre

Near infrared survey of the nuclear region of the Milky Way

G.J. Madsen, L.M. Haffner, R.J. Reynolds

AST/RO sub-mm survey of the galactic center

C.L. Martin, W.M. Walsh, K. Xiao and 5 co-authors

Preliminary results from an open clusters polarimetric survey

A.M. Orsatti, M.M. Vergne, C. Feinstein, R.E. Martinez

The Guide Star Catalog II. Properties of the GSC 2.3 release

A. Spagna, M.G. Lattanzi, B. McLean and 9 co-authors

First brown dwarfs from the UKIRT Infrared Deep Sky Survey (UKIDSS)

R. Tata, E.L. Martin, T. Kendall and 3 co-authors

Kinematics of nearby $\mathrm{K}-\mathrm{M}$ dwarfs: first results

A.R. Upgren, R.P. Boyle, J. Sperauskas, S. Bartašiūtè

\subsection{New calibrations and models}

Estimating interstellar extinction toward to elliptical galaxies and star clusters

E.B. de Amôres and J.R.D. Lépine

Using the genetic algorithms to study the galactic structure

E.B. de Amôres, A.C. Robin

DSS-I value added catalog of stellar parameters and the SEGUE pipeline

T.C. Beers, Y. Lee, T. Sivarani, C. Allende Prieto, R. Wilhelm, P. Re Fiorentin, C. Bailer-Jones, J.E. Norris, and the SEGUE Calibration Team

All-sky counts in Gaia's unfiltered passband

R. Drimmel, A. Spagna, B. Bucciarelli, M. Lattanzi, R. Smart 
Chemical abundances in the ancient Milky Way: G-type SDSS stars Automated determination of $\mathbf{T}_{\text {eff }}, \log g,[\mathrm{Fe} / \mathrm{H}]$ and $[\alpha / \mathrm{Fe}]$.

P. Girard, C. Allende Prieto, C. Soubiran

Search for and investigation of new open clusters using the data from huge astronomical catalogues

S. Koposov, E. Glushkova

Classification of eclipsing binaries in large surveys

O. Malkov, E. Oblak

The Strömvil photometric system: classifying faint stars

A.G.D. Philip, R.P. Boyle

A library of synthetic galaxy spectra for GAIA: comparison with SDSS

P. Tsalmantza, M. Kontizas, R. Korakitis, and 8 co-authors

\subsection{Exploiting existing surveys}

Using the Geneva-Copenhagen survey to study the nature of the Hyades stream

B. Famaey, F. Pont, X. Luri, S. Udry, M. Mayor, A. Jorissen

VIMOS@VLT photometric and spectroscopic survey of the Sagittarius dwarf spheroidal galaxy

G. Giuffrida, S. Zaggia, C. Izzo and 6 co-authors

Investigation of star clusters detected automatically in 2MASS Point Source Catalogue

E. Glushkova, S. Koposov

Searching for White Dwarfs in Surveys

A. Kawka, S. Vennes

Short timescale variability in the faint sky variability survey

L. Morales-Rueda, P.J. Groot, T. Augusteijn, G. Nelemans, P.M. Vreeswijk, E.J.M. van den Besselaar

The White Dwarf Population in IPHAS

L. Morales-Rueda, P.J. Groot, R. Napiwotzki, J.E. Drew

Variable stars in the MOA database

L. Skuljan, I.A. Bond

The RAVE survey: Using the local escape velocity to determine the mass of the Milky Way

M.C. Smith, G.R. Ruchti, A. Helmi, R.F.G. Wyse, and the RAVE collaboration

Kinematics of nearby disk stars from Hipparcos database

R. Teixeira, R.E. de Souza

\subsection{Future survey projects}

JASMINE-astrometric map of the galactic bulge

N. Gouda, Y. Kobayashi, Y. Yamada and 12 co-authors

Vista variables in the Via Lactea (VVV)

D. Minniti, P. Lucas, A. Ahumada and 55 co-authors

LOBSTER telescopes as X-ray All Sky Monitors

R. Hudec, M. Skulinova, L. Pina, L. Sveda

A very small astrometry satellite mission: Nano-JASMINE

Y. Kobayashi, N. Gouda, T. Tsujimoto

Development of a very small telescope for space astrometry surveyer

M. Suganuma, Y. Kobayashi, N. Gouda and 4 co-authors 
A near-infrared high-resolution spectroscopic survey of Galactic bulge stars

T. Tsujimoto, N. Kobayashi, Y. Ikeda and 5 co-authors

New method for astrometric measurements in Space Mission, JASMINE

T. Yano, N. Gouda, Y. Yamada

JASMINE simulator

Y. Yamada, N. Gouda, T. Yano and 7 co-authors

\section{Discussion and conclusions}

Reported by Christopher J. Corbally

\subsection{The panel discussion}

About an hour was set aside to ensure that this meeting truly was a joint discussion. With the speakers seated in front, questions and comments on large surveys in the Galaxy were invited.

A first question concerned how detailed should be the information on abundances. It was concluded that first the overall $\alpha$-element abundances should be determined but that individual element abundances, since they have different nucleosynthesis origins, should certainly be investigated subsequently.

This invoked a reminder that surveys were useful for finding not only the very low metallicity stars, but also stars with higher than solar abundances. The larger the surveys, the more likely to find such stars. Large surveys would also have the best chance of finding the very rare types of peculiar stars, but to do this effectively auto-classification tools needed further refinement.

The discussion then turned to the advantages forthcoming in the larger surveys, those of the order of 100 s of millions of stars, with radial velocity as well as full photometric and spectroscopic data, about which the participants had been hearing earlier in the morning (Session IV). These promised improved calibrations, and so improvement in derived parameters and the understanding of stellar atmospheres and convective modeling. However, if these surveys were to fulfill their promise, the adequate modeling of such data was needed, as is already recognized. It was agreed that critical components for such modeling were the Galactic bulge and arms. There was a prediction that, when we have data on a billion stars, we shall realize that the Milky Way is a dynamical, not an equilibrium system.

It was important, when thinking of the large surveys, to recognize niches that they could not fill. One such was a survey with high sensitivity to low metallicities, i.e., the SkyMapper project at Siding Spring Observatory, and another was the analysis of proper motion survey catalogs for data on wide binaries, which can be cleanly classified as to Galactic population.

One aspect of Galactic astronomy could not be well covered in the time available for the Joint Discussion; this was radio data, and so the role of molecular clouds and gas dynamics within the Milky Way. Clearly a further, longer meeting on large surveys and the Galaxy would be needed in the not-too-distant future.

So, the panel discussion served to solidify concepts presented by the speakers during the JD13, to broaden horizons, and to enhance the sense of common enterprise and excitement as all looked forward to exploiting the really large databases for research into Galactic astronomy.

\subsection{Tim de Zeeuw: Concluding Remarks}

In understanding the formation of galaxies, the Milky Way has the unique advantage of having a 'fossil record' that can be read in detail, since we have access to billions 
of individual stars. The Milky Way is also an average galaxy, and so provides 'nearfield' cosmology and a testing ground for the paradigm of formation through hierarchical merging of many small building blocks. So specific questions need to be asked of the Milky Way: $(i)$ when its stars formed; (ii) when and how it was assembled; and (iii) how its dark matter is distributed. These are indeed the questions which many ongoing and planned surveys address and which have been reviewed in this Joint Discussion.

Ongoing surveys, including 2MASS and SDSS/SEGUE, reveal many coherent structures in the Galactic halo, such as tidal tails. These are targets for photometric surveys coming on line (Pan-STARRS, AKARI, VST/VISTA) and those planned, if not yet funded (Pan-STARRS 4 and LSST). From thousands to multiple millions of radial velocities have issued from present surveys (Geneva/Copenhagen, SDSS/SEGUE, RAVE) and are anticipated in the future (Gaia), while the impact of the astrometric work of Hipparcos has stimulated both work from the ground and the planning of future missions (Gaia, and Japanese and US missions). Such photometric, kinematic and spectroscopic surveys are providing the all-important stellar parameters that input into Galactic structure information.

There is mounting evidence for different kinematic groups with very homogeneous elemental abundances within the group, but distinct difference between groups. Such evidence is found not only in the stellar halo but now also in the thick disk, and it is very suggestive of successive merging of separate building blocks. These building blocks appear not to be the same as the precursors of the dwarf spheroidals that we observe around the Milky Way today.

The large data sets from ongoing and future surveys promise to unravel at least part of not only the Milky Way formation history but also of the Local Group history, if in less detail. However, these surveys face some significant challenges. Photometric calibration is critical and the derivation of stellar parameters for all classes of stars has to be approached cautiously. Correcting for extinction will be important in some directions and can be ignored in others, while astrometric parallaxes must be of sufficient precision as to give independent distances. The additional physical information provided by variable stars needs multi-epoch observations in a well-planned cadence, something more easily achieved from the ground than from spinning satellites. The input of all these data into the dynamical modeling of the Galaxy will have massive discriminating power between the various formation scenarios, but only if the existing computational machinery for this is developed considerably to deal with the immense data sets which are foreseen.

If there are challenges for surveys, there are also opportunities. Ground-based surveys, e.g., RAVE, can find much complementarity with space-based surveys, e.g., the all-sky Gaia. The complementarity is both in providing a pilot project to pave the way for a fainter, space probe, and also in eventually going fainter than the space mission limit. The space-based photometry can also help calibrate the ground-based measurements. Japanese space astrometry plans focus on an infrared study of the Bulge, certainly a complement to the reach of Gaia which is confined there to the low-extinction windows. High-resolution spectroscopic follow-ups, e.g., with the proposed WFMOS for Subaru, are going to be essential complements to space-based data, and perhaps even more such projects should be developed.

In conclusion, there is much to look forward to in the next decade for our understanding of the formation of the Milky Way and Local Group.

\section{Acknowledgements}

While the Scientific Organizing Committee's preparations were essential to this meeting, its obvious success owes much to the considerable effort and enthusiasm contributed 
by the speakers, by the poster presenters, and by those who attended. We thank them and their various granting agencies wholeheartedly.

\section{References}

Allende Prieto, C., \& Lambert, D. L. 1999, A\& A, 352, 555

Alonso, A., Arribas, S. \& Martinez-Roger, C. 1996, A\& $A, 313,873$

Bailer-Jones, C. A. L. 2002, in: R. Gupta, H. P. Singh \& C. A. L. Bailer-Jones (eds.), Automated Data Analysis in Astronomy (New Delhi, London: Narosa Pub. House), p. 83

Bailer-Jones, C. A. L., Irwin, M., \& von Hippel, T. 1998, MNRAS, 298, 361

Jordi, C., Høg, E., Brown, A. G. A., et al. 2006, MNRAS, 367, 290

Marshall, D. J., Robin, A. C., Reylé, C., Schultheis, M., Picaud, S. 2006, A\&AA, 453, 635

Nishiyama, S., Nagata, T., Kusakabe, N., et al. 2006, ApJ, 638, 839

Nordström, B., Mayor, M., Andersen, J., et al. 2004, A\&\&A, 418, 989

Olsen, E. H. 1988, A\& A, 189, 173

Snider S., Allende Prieto, C., von Hippel, T., et al. 2001, ApJ, 562, 528 